\title{
Renormalization group domains of the scalar Hamiltonian
}

\author{
C.Bagnuls ${ }^{1}$, C.Bervillier ${ }^{2}$ \\ 1 Service de Physique de l'Etat Condensé, \\ CE Saclay, F91191 Gif-sur-Yvette Cedex, France \\ 2 Service de Physique Théorique, \\ CE Saclay, F91191 Gif-sur-Yvette Cedex, France
}

Received March 9, 2000

Using the local potential approximation of the exact renormalization group (RG) equation, we show various domains of values of the parameters of the $O(1)$-symmetric scalar Hamiltonian. In three dimensions, in addition to the usual critical surface $S_{\text {c }}$ (attraction domain of the Wilson-Fisher fixed point), we explicitly show the existence of a first-order phase transition domain $S_{\mathrm{f}}$ separated from $S_{\mathrm{c}}$ by the tricritical surface $S_{\mathrm{t}}$ (attraction domain of the Gaussian fixed point). $S_{\mathrm{f}}$ and $S_{\mathrm{c}}$ are two distinct domains of repulsion for the Gaussian fixed point, but $S_{\mathrm{f}}$ is not the basin of attraction of a fixed point. $S_{\mathrm{f}}$ is characterized by an endless renormalized trajectory lying entirely in the domain of negative values of the $\varphi^{4}$-coupling. This renormalized trajectory also exists in four dimensions making the Gaussian fixed point ultra-violet stable (and the $\varphi_{4}^{4}$ renormalized field theory asymptotically free but with a wrong sign of the perfect action). We also show that a very retarded classical-to-lsing crossover may exist in three dimensions (in fact below four dimensions). This could be an explanation of the unexpected classical critical behaviour observed in some ionic systems.

Key words: renormalization group, critical, tricritical, first-order phase transition, crossover

PACS: 05.10.Cc, 05.70.Jk, 11.10.Hi, 61.20.Qg

\section{Introduction}

The object of this paper ${ }^{1}$ is to carry on studying the local potential approximation of the exact renormalization group ( $R G$ ) equation for the scalar theory [1]. In the previous publication [2] (to be considered as part I of the present work), we had already considered this approximation with a view to qualitatively discuss the connection between the standard perturbative renormalization of field theory (as it

\footnotetext{
${ }^{1}$ Based on a talk given at "RG 2000", Taxco, Mexico, January 1999
} 
can be found in most textbooks on field theory, see for example [3]) and the modern view [4] in which the renormalized parameters of a field theory are introduced as the "relevant" directions of a fixed point (FP) of a RG transform. Actually the local potential approximation, which allows us to consider all the powers of the field $\varphi$ on the same footing, is an excellent textbook example of the way in which infinitely many degrees of freedom are accounted for in (nonperturbative) RG theory. Almost all the characteristics of the $\mathrm{RG}$ theory are involved in this approximation. The only lacking features are related to phenomena highly correlated to the non-local parts neglected in the approximation and, when the critical exponent $\eta$ is small (especially for $d=4$ and $d=3$ ), one expects the approximation to be qualitatively correct regarding all aspects of the RG theory [1].

In the following we look at the domains of attraction or of repulsion of fixed points in the $O(1)$ scalar theory in three and four dimensions $(d=3$ and $d=4)$.

At first sight, one could think that the issue considered is very simple since, with regard to criticality, the $O(1)$-symmetric systems in three dimensions are known to belong to the same class of universality (the Ising class). Now, because the Ising class is associated to the domain of attraction of the unique (non-trivial) WilsonFisher fixed point [5], then by adjusting one parameter (in order to reach the critical temperature $^{2}$ ) any $O(1)$ scalar Hamiltonian should be driven to the Wilson-Fisher fixed point under the action of renormalization. Consequently there would be only two domains for the $O(1)$ scalar theory: the critical subspace $S_{\text {c }}$ (of codimension 1 ) in the Wilson space $(S)$ of infinite dimensions of the Hamiltonian parameters (in which the RG transforms generate flows) and the complement to $S$ of $S_{\mathrm{c}}$ (corresponding to noncritical Hamiltonians).

In fact, this is not correct because there is another fixed point in $S$ : the Gaussian fixed point which, although trivial, controls tricritical behaviours in three dimensions. Now each FP has its own basin of attraction in $S$ [5]. The attraction domain of the Gaussian FP is the tricritical subspace $S_{\mathrm{t}}$ of codimension 2 (with no intersection with $S_{\mathrm{c}}$ ). In addition, we show that there is a second subspace of codimension 1 in $S$, called $S_{\mathrm{f}}$, which is different from $S_{\mathrm{c}}$, and thus which is not a domain of attraction to the Wilson-Fisher fixed point. There is no FP to which a point of $S_{\mathrm{f}}$ is attracted. $S_{\mathrm{f}}$ is characterized by a negative sign of the $\varphi^{4}$-Hamiltonian parameter $u_{4}$ and is associated with systems undergoing a first-order phase transition [6]. We show that an endless attractive RG trajectory is associated to this domain of first-order transitions. It is a renormalized trajectory (denoted below by $\mathrm{T}_{1}^{\prime \prime}$ ) that emanates from the Gaussian fixed point. The frontier between $S_{\mathrm{f}}$ and $S_{\mathrm{c}}$ corresponds to the tricritical subspace $S_{\mathrm{t}}$ which is the domain of attraction of the Gaussian fixed point while $S_{\mathrm{f}}$ and $S_{\mathrm{c}}$ are two distinct domains of repulsion for the Gaussian fixed point.

Actually, the situation is in conformity with the usual view. Considering the famous $\varphi^{4}$-model [Landau-Ginzburg-Wilson (LGW) Hamiltonian] in which the associated coupling $u_{4}$ is positive, the Hamiltonian at criticality is attracted exclusively to the Wilson-Fisher fixed point, but if $u_{4}$ is negative, a $\varphi^{6}$-term is required for sta-

\footnotetext{
${ }^{2}$ We assume that the second relevant field, corresponding to the magnetic field for magnetic systems, is set equal to zero.
} 
bility, but then one may get either a tricritical phase transition or a second- or a first-order transition [7]. In the present study we do not truncate the Hamiltonian which involves all the powers of the field $\varphi$.

We explicitly show that a system which would correspond to an initial point lying very close to the frontier $S_{\mathrm{t}}$ in the critical side (in $S_{\mathrm{c}}$ ) would display a retarded classical-to-Ising crossover [8]. This result is interesting with regard to ionic systems (for example) in which a classical behaviour has been observed while an Ising-like critical behaviour was expected. The eventuality of a retarded crossover from the classical to the Ising behaviour has previously been mentioned but without explaining theoretically how this kind of crossover could develop [9]. In [8] a calculation suggests that the RPM model for ionic systems would specifically correspond to a scalar Hamiltonian with a negative sign for the $\varphi^{4}$-Hamiltonian parameter (but the order parameter chosen is not the bulk density [10]). This calculation has motivated the present study.

We also indicate that the renormalized trajectory $\mathrm{T}_{1}^{\prime \prime}$ still exists in four dimensions. This makes the Gaussian fixed point ultraviolet stable and the scalar field theory formally asymptotically free. However the associated "perfect" action [11] would have the wrong sign to provide us with an acceptable (well defined) field theory.

The paper is arranged as follows. In section 2 we briefly present the local potential approximation of the exact $\mathrm{RG}$ equation to be studied. We introduce the strategy we have chosen to solve the resulting nonlinear differential equation with a view to show the trajectories of interest in the space $S$ of infinite dimension. Because the practical approach to the Gaussian fixed point is made difficult due to the logarithmic slowness characteristic of a marginally irrelevant direction (for $d=3$ ), we found it useful to first test our numerical method with a close approach to the Wilson-Fisher fixed point. We present the characteristic results of this approach and various kinds of domains corresponding to $u_{4}>0$ (a kind of a summary of [2])

In section 3 we describe various kinds of attraction or repulsion domains of the Gaussian fixed point (for a negative value of the $\varphi^{4}$-Hamiltonian parameter) corresponding to tricritical, critical and first-order subspaces. Then we discuss the consequences and especially explicitly show how a retarded crossover from the classical to the Ising behaviour can be obtained.

We then shortly discuss the case $d=4$ when $u_{4}<0$.

In two appendices we report on some technical aspects of the numerical treatment of the RG equation studied, in particular on the appearing of spurious nontrivial tricritical fixed points (appendix A.1).

\section{The RG equation studied}

The local potential approximation was first considered by Nicoll et al. [12] from the sharp cutoff version of the exact RG equation of Wegner and Houghton [13]. It was rederived by Tokar [14] by using approximate functional integrations and rediscovered by Hasenfratz and Hasenfratz [15]. As in [2] we adopt the notation of 
the latter authors and consider the following nonlinear differential equation for the simple function $f(l, \varphi)$ :

$$
\dot{f}=\frac{K_{d}}{2} \frac{f^{\prime \prime}}{1+f^{\prime}}+\left(1-\frac{d}{2}\right) \varphi f^{\prime}+\left(1+\frac{d}{2}\right) f
$$

in which a prime refers to a derivative with respect to the constant dimensionless field $\varphi$ (at constant $l$ ) and $f(l, \varphi)=V^{\prime}(l, \varphi)$ is the derivative of the dimensionless potential $V(l, \varphi) ; \dot{f}$ stands for $\partial f / \partial l_{\varphi}$ in which $l$ is the scale parameter defined by $\Lambda / \Lambda_{0}=\mathrm{e}^{-l}$ and corresponding to the reduction to $\Lambda$ of an arbitrary initial momentum scale of reference $\Lambda_{0}$ (the initial sharp momentum cutoff). Finally, $K_{d}$ is the surface of the $d$-dimensional unit sphere divided by $(2 \pi)^{d}$.

A fixed point is a solution of the equation $\dot{f}=0$. The study of the resulting second order differential equation provides the following results:

- $d \geqslant 4$, no FP is found except the Gaussian fixed point.

- $3 \leqslant d<4$, one nontrivial FP (the Wilson-Fisher fixed point [5]) is found $[15-17]$.

- A new nontrivial FP emanates from the origin (the Gaussian fixed point) below each dimensional threshold $d_{k}=2 k /(k-1), k=2,3, \ldots, \infty[18]$.

If one represents the function $f(l, \varphi)$ as a sum of monomials of the form:

$$
f(l, \varphi)=\sum_{n} u_{2 n}(l) \varphi^{2 n-1}
$$

then, for $d=3$, the Wilson-Fisher fixed point $f^{*}$ is located in $S$ at $[2]: u_{2}^{*}=$ $-0.461533 \cdots, u_{4}^{*}=3.27039 \cdots, u_{6}^{*}=14.4005 \cdots, u_{8}^{*}=32.31289 \cdots$, etc.

Once the FP is known, one may study its vicinity which is characterized by orthogonal directions corresponding to the infinite set of eigenvectors, solutions of the differential equation (2.1) linearized at $f^{*}$. The eigenvectors associated to positive eigenvalues are said to be relevant; when the eigenvalues are negative they are said to be irrelevant and marginal otherwise [19].

The relevant eigenvectors correspond to directions along which the RG trajectories go away from the FP and the irrelevant eigenvectors correspond to directions along which the trajectories go into the FP. A marginal eigenvector may be relevant or irrelevant.

Our present FP $f^{*}$ has only one relevant direction and infinitely many irrelevant directions (no marginal direction, still see [1]). As already explained and shown in [2], in order to approach $f^{*}$ starting from an initial point in $S$, one must adjust one parameter of the initial function $f(0, \varphi)$. This amounts to fixing the temperature of a system to its critical temperature.

Starting with a known initial function (at "time" $l=0$ ) say:

$$
f(0, \varphi)=u_{2}(0) \varphi+u_{4}(0) \varphi^{3}
$$


we adjust $u_{2}(0)$ to the critical value $u_{2}^{\mathrm{c}}=-0.29958691 \cdots$ corresponding to $u_{4}(0)=$ 3 so that $f(l, \varphi)$ [solution at time $l$ of the differential equation (2.1)] approaches $f^{*}$ when $l \rightarrow \infty$. The approach to $f^{*}$ is characterized by the least negative eigenvalue $\lambda_{2}=-1 / \omega_{1}\left(\omega_{1}\right.$ was noted $\omega$ in [2]). This means that, in the vicinity of $f^{*}$ any parameter $u_{n}(l)$ evolves as follows $(l \rightarrow \infty)$ :

$$
u_{n}(l) \simeq u_{n}^{*}+a_{n} \exp \left(-\omega_{1} l\right) .
$$

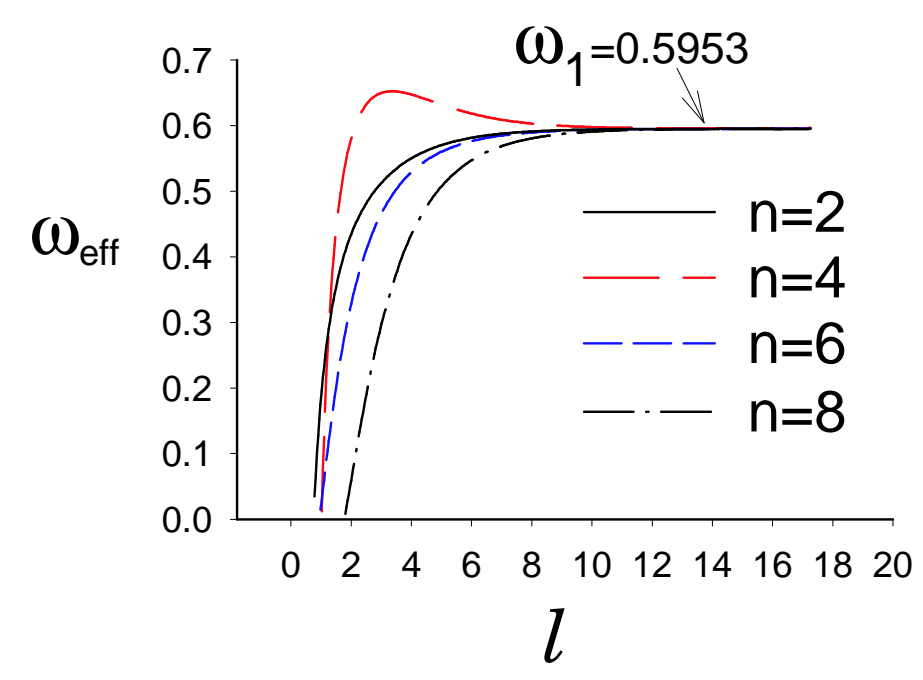

Figure 1. Evolutions for $d=3$ of the first four Hamiltonian parameters $u_{2}(l)$, $u_{4}(l), u_{6}(l), u_{8}(l)$ in a close approach to the Wilson-Fisher fixed point $f^{*}$ along $\mathrm{T}_{1}$ or $\mathrm{T}_{1}^{\prime}$. The effective inverse eigenvalue $\omega_{\text {eff }}(l)$ is given by equation $(2.2)$ for $n=2,4,6,8$. All these quantities reach the same universal value $\omega_{1}$ characteristic of the least irrelevant eigendirection of $f^{*}$. To get this close approach to $f^{*}$ from equation (2.1), the initial critical value $u_{2}^{\mathrm{c}}$ corresponding to $u_{4}(0)=3$, has been determined with more than twenty digits.

Figure 1 illustrates this feature for the first four $u_{n}(l)$ 's in the approach to $f^{*}$. In [2] the two associated attractive trajectories (locally tangent to the least irrelevant eigenvector in the vicinity of $f^{*}$ ) were noted $T_{1}$ and $T_{1}^{\prime}$.

One may also constrain the trajectory to approach $f^{*}$ along the second irrelevant direction (with the associated attractive trajectories noted $\mathrm{T}_{2}$ or $\mathrm{T}_{2}^{\prime}$ in [2] and associated with the second least negative eigenvalue $\left.\lambda_{3}=-1 / \omega_{2}\right)$. In this case a second parameter of the initial $f$ must be adjusted, e.g., $u_{4}(0)$ must be adjusted to $u_{4}^{\mathrm{c}}$ and simultaneously $u_{2}(0)$ to the corresponding $u_{2}^{\mathrm{c}}$, see [16,2]. Then, in the vicinity of $f^{*}$, any parameter $u_{n}(l)$ will evolve as follows:

$$
u_{n}(l) \simeq u_{n}^{*}+a_{n}^{\prime} \exp \left(-\omega_{2} l\right) .
$$

Looking for this kind of approach to $f^{*}$, we have found that $6.66151663<u_{4}^{\mathrm{c}}<$ 6.66151669 and $u_{2}^{\mathrm{c}}=-0.58328898880579 \cdots$ This has allowed us to estimate $\omega_{2} \cong$ 2.84. Although the shooting method is certainly not well adapted to determining 


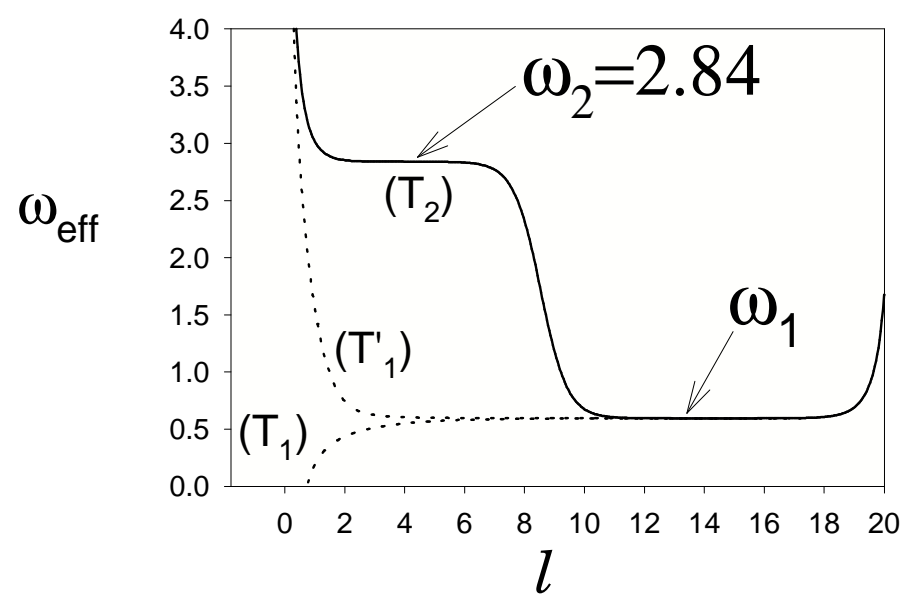

Figure 2. When a second condition is imposed on the initial Hamiltonian parameters, the approach to $f^{*}$ may be adjusted such as to asymptotically take the second least irrelevant eigendirection. Here $\omega_{\text {eff }}(l)$ is given by equation $(2.2)$ for $n=2$ it clearly undergoes (full line) a flat inflection point at the value $\omega_{2}=2.84$ corresponding to an approach to $f^{*}$ along $\mathrm{T}_{2}$, the greater the critical parameter $u_{4}^{\mathrm{c}}$ is accurately determined the longer is the flat extremum. Because $u_{4}^{\mathrm{c}}$ is not completely determined [within the available accuracy in solving equation (2.1)] the trajectory leaves the direction of $\mathrm{T}_{2}$ to take one of the two directions of approach associated to the least irrelevant inverse eigenvalue $\omega_{1}$ (corresponding to $\mathrm{T}_{1}$ or $\mathrm{T}_{1}^{\prime}$ as indicated by dashed curves). Here, the trajectory corresponding to the full line goes along $\mathrm{T}_{1}^{\prime}$. Again a flat extremum of $\omega_{\text {eff }}(l)$ indicates the approach along an eigenvector of $f^{*}$ and requires an accurate determination of the critical value $u_{2}^{\mathrm{c}}$. Because this determination is not complete, the trajectory ends up going away from $f^{*}$ as indicated by the sudden departure of $\omega_{\text {eff }}(l)$ from $\omega_{1}$ for the large values of $l$.

the eigenvalues (see the huge number of digits required in determining $u_{2}^{\mathrm{c}}$ and $u_{4}^{\mathrm{c}}$ ), our estimate is close to $\omega_{2} \cong 2.8384$ found by Comellas and Travesset [20].

Because $u_{4}^{\mathrm{c}}$ cannot be perfectly determined, the trajectory leaves the trajectory $\mathrm{T}_{2}$ before reaching $f^{*}$ to take one of the two directions $\mathrm{T}_{1}$ or $\mathrm{T}_{1}^{\prime}$ (corresponding to $\left.\omega_{1}\right)$. Figure 2 illustrates this effect with the evolution, for $n=2$, of the following effective eigenvalue:

$$
\omega_{\text {eff }}^{(n)}(l)=-\frac{\mathrm{d}^{2} u_{n}(l) / \mathrm{d} l^{2}}{\mathrm{~d} u_{n}(l) / \mathrm{d} l}
$$

the definition of which does not refer explicitly to $f^{*}$. The evolution of $\omega_{\text {eff }}(l)$ shows a flat extremum (or a flat inflection point) at an RG eigenvalue of $f^{*}$ each time the RG flow runs along an eigendirection in the vicinity of $f^{*}$.

Similar to $u_{4}^{\mathrm{c}}$, the value $u_{2}^{\mathrm{c}}$ cannot be perfectly determined. Consequently, the trajectory ends up going away from the fixed point. This provides us with the opportunity of determining the only positive (the relevant) eigenvalue corresponding to the critical exponent $\nu=\lambda_{1}=-1 / \omega_{0}\left[\omega_{\text {eff }}(l)\right.$ shows then a flat extremum at $\omega_{0}$ when the flow still runs in close vicinity of $\left.f^{*}\right]$. Finally, far away from the fixed point, 


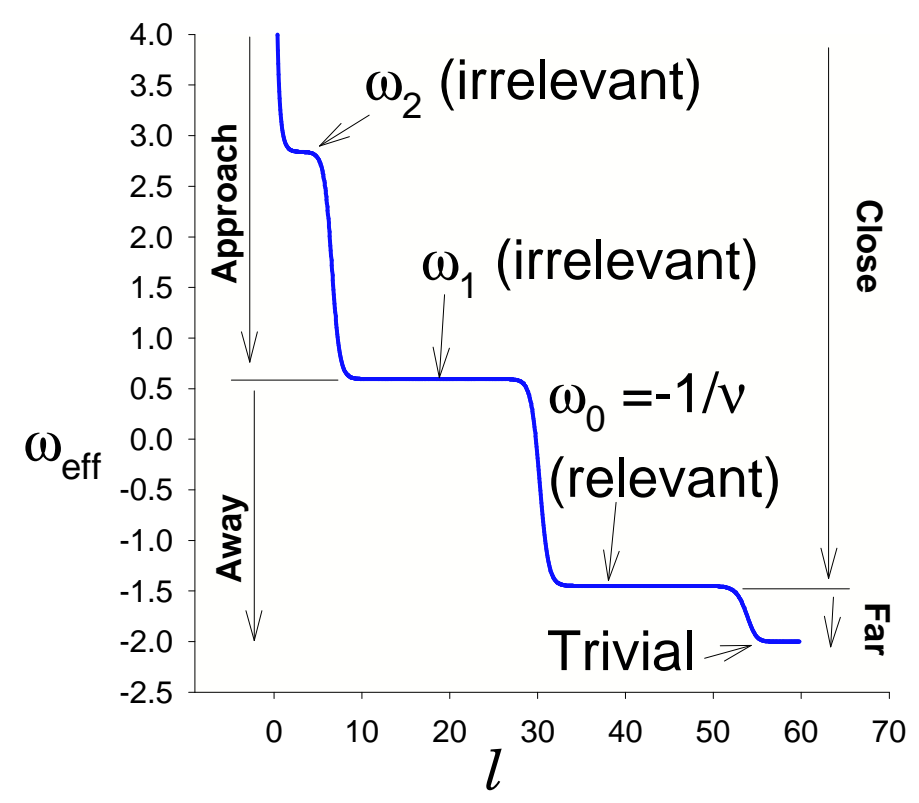

Figure 3. This figure is a continuation of figure 2. It shows the various plateaux that $\omega_{\text {eff }}(l)$ undergoes along a RG trajectory first adjusted to approach $f^{*}$ along the second irrelevant direction (plateau at $\omega_{2}=2.84$ ). Because it is not possible to exactly determine the initial conditions, the trajectory always ends up going away from the fixed point towards the trivial high temperature fixed point characterized by the classical value $\frac{1}{2}$ (for minus the inverse of $\omega_{\text {eff }}(l)$, thus the final plateau at -2 ). In-between, the RG flow has been influenced by the close vicinity of the least irrelevant eigenvector (plateau at $\omega_{1}$ ) and that of the relevant eigenvector (plateau at $\omega_{0}=-\frac{1}{\nu}$ ). The various regimes of the RG flows are indicated by the vertical arrows on the left (direction of the flow with respect to the fixed point) and on the right of the figure (distance to the fixed point).

the RG trajectory approaches the trivial high temperature fixed point characterized by a classical eigenvalue (equal to $\frac{1}{2}$ ). The global picture summarizing the evolution of $\omega_{\text {eff }}(l)$ along the RG trajectory initialized in such a way as to approach $f^{*}$ first along $\mathrm{T}_{2}$, is drawn in figure 3 .

The values we have determined by this shooting method are (for eigenvalues other than the already mentioned $\left.\omega_{2}\right)$ :

$$
\begin{aligned}
\omega_{1} & \cong 0.5953, \\
\nu & \cong 0.68966,
\end{aligned}
$$

which are close to the values found, for example, in $[15,20]: \omega_{1} \cong 0.5952$ and $\nu \cong$ 0.6895 .

\section{Trajectories for $u_{4}<0$}

In the preceding section, we have obtained a RG trajectory approaching the Wilson-Fisher fixed point $f^{*}$ along $\mathrm{T}_{2}$ by adjusting two parameters of the initial 
Hamiltonian $\left(u_{4}^{\mathrm{c}}\right.$ and $\left.u_{2}^{\mathrm{c}}\right)$. This is exactly the procedure one must follow to determine a tricritical RG trajectory approaching the Gaussian fixed point in three dimensions (because of its two relevant directions). The only difficulty is to discover initial points in $S$ which are attracted to the Gaussian fixed point. To this end, we again use the shooting method.

Based on the usual arguments regarding the LGW Hamiltonian as well as taking into account the work done by Aharony on compressible ferromagnets [6], one expects to find the tricritical surface in the sector $u_{4}<0$ (and with $u_{2}>0$ ). Thus we have tried to approach the Gaussian fixed point starting with initial function $f(0)$ of the form:

$$
f(0, \varphi)=u_{2}(0) \varphi+u_{4}(0) \varphi^{3}+u_{6}(0) \varphi^{5}
$$

with (not large) negative values of $u_{4}(0)$, for example $u_{4}(0)=-1$.

Because the Gaussian fixed point is twice unstable, we must adjust two parameters to approach it starting with (3.1). We do that by successive tries (shooting method). For example, if we choose $u_{4}(0)=-1$ and $u_{6}(0)=3$ and determine a value of $u_{2}(0)$ such as to get a trajectory which does not go immediately towards the trivial high temperature fixed point, the best we obtain is a trajectory which approaches the Wilson-Fisher fixed point (thus the corresponding initial point belongs to the attraction domain of $f^{*}$ although $\left.u_{4}(0)<0[6]\right)$. But if $u_{6}(0)=2$, the adjustment of $u_{2}(0)$ with a view to counterbalance the effect of the most relevant direction of the Gaussian fixed point (which would drive the trajectory toward the high temperature FP) yields a runaway RG flow towards larger and larger negative values of $u_{4}(l)$. From now on, the target is bracketed: the tricritical trajectory corresponding to $u_{4}(0)=-1$ can be obtained with a value of $u_{6}(0)$ in the range ]2, 3[ (we actually find a rather close approach to the Gaussian fixed point for $2.462280>u_{6}(0)>2.4622788$ and $\left.6.4618440 \cdots>u_{2}(0)>6.4618407 \cdots\right)$.

In order to understand the origin of the direction of runaway in the sector of negative values of $u_{4}$, it is worth studying the properties of the Gaussian fixed point by linearization of the $R G$ flow equation in the vicinity of the origin. If we request the effective potential to be bounded by polynomials then the linearization of equation (2.1) is identified with the differential equation of Hermite's polynomials of degree $n=2 k-1$ for the set of discrete values of $\lambda$ satisfying [15]:

$$
\frac{2+d-2 \lambda_{k}}{d-2}=2 k-1 \quad k=1,2,3, \ldots
$$

from which it follows that

- for $d=4: \lambda_{k}=4-2 k, k=1,2,3, \ldots$, there are two non-negative eigenvalues: $\lambda_{1}=2$ and $\lambda_{2}=0$

- for $d=3: \lambda_{k}=3-k, k=1,2,3, \ldots$, there are three non-negative eigenvalues: $\lambda_{1}=2, \lambda_{2}=1$ et $\lambda_{3}=0$.

If we denote by $\chi_{k}(\varphi)$ the eigenfunctions associated to the eigenvalue $\lambda_{k}$, it comes: 


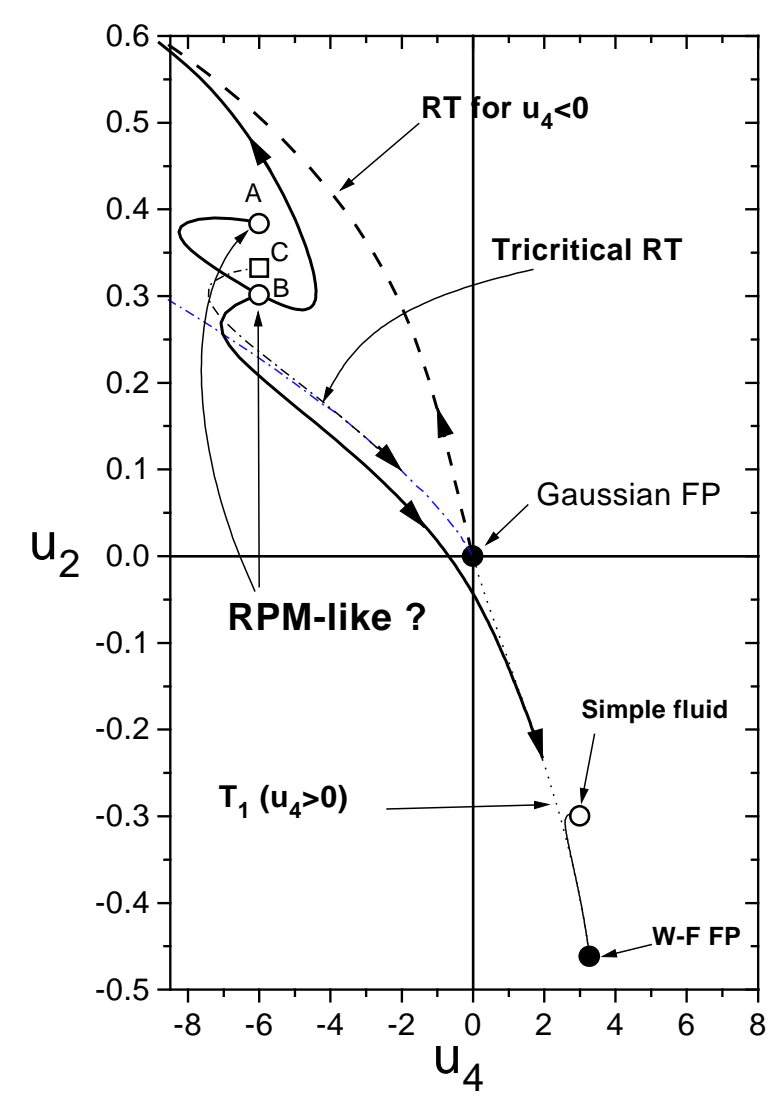

Figure 4. Domains of attraction and repulsion of the Gaussian fixed point. The figure represents projections onto the plane $\left\{u_{2}, u_{4}\right\}$ of various RG trajectories running in the space $S$ minus one dimension. The flows have been obtained by solving equation (2.1). Black circles represent the Gaussian and the Wilson-Fisher (W-F FP) fixed points. The arrows indicate the directions of the RG flows on the trajectories. The ideal trajectory (dot line) which interpolates between these two fixed points represents the usual renormalized trajectory $\mathrm{T}_{1}$ corresponding to the so-called $\varphi_{3}^{4}$ renormalized field theory in three dimensions (usual RT for $\left.u_{4}>0\right)$. White circles represent the projections onto the plane of initial critical Hamiltonians. For $u_{4}(0)>0$, the effective Hamiltonians run toward the Ising fixed point asymptotically along $\mathrm{T}_{1}$ (simple fluid). Instead, for $u_{4}(0)<0$ and according to the values of Hamiltonian coefficients of higher order $\left(u_{6}, u_{8}\right.$, etc.), the RG trajectories either (A) meet an endless RT emerging from the Gaussian FP (dashed curve) and lying entirely in the sector $u_{4}<0$ or (B) meet the usual $\mathrm{RT} \mathrm{T}_{1}$ to reach the Ising fixed point. The frontier which separates these two very different cases (A and B) corresponds to initial Hamiltonians lying on the tricritical subspace $S_{\mathrm{t}}$ (white square C). This is a source of RG trajectories flowing asymptotically toward the Gaussian FP along the tricritical RT. Notice that the coincidence of the initial point $B$ with the $R G$ trajectory starting at point $A$ is not real (it is accidental, due to a projection onto a plane of trajectories lying in a space of infinite dimension). The points A or B could correspond to the restricted primitive model of ionic systems (see [8]). 
- $\chi_{1}^{+}=\varphi, \chi_{2}^{+}=\varphi^{3}-\frac{3}{2} \varphi, \chi_{3}^{+}=\varphi^{5}-5 \varphi^{3}+\frac{15}{4} \varphi, \ldots$, whatever the spatial dimensionality $d$.

The superscript "+" is just a reminder of the fact that the eigenfunctions are defined up to a global factor and thus the functions $\chi_{k}^{-}(\varphi)=-\chi_{k}^{+}(\varphi)$ are also eigenfunctions with the same eigenvalue $\lambda_{k}$.

\subsection{Case $d=3$}

Similar to $\chi_{2}^{+}$, the direction provided by $\chi_{2}^{-}$in $S$ is a direction of instability of the Gaussian fixed point. Now $\chi_{2}^{+}$is associated with the well known renormalized trajectory $T_{1}$ on which the usual (massless) $\varphi_{3}^{4}$-field theory [16,2] is defined, for the same reasons a renormalized trajectory $\mathrm{T}_{1}^{\prime \prime}$ locally tangent to $\chi_{2}^{-}$in the vicinity of the origin of $S$ exists with the same properties as $\mathrm{T}_{1}$ (see [2]). The difference is that $\mathrm{T}_{1}^{\prime \prime}$ lies entirely in the sector $u_{4}<0$ and is endless (not ended by a fixed point).

This endless renormalized trajectory is associated with systems undergoing a first-order phase transition. This is due to the absence of fixed point [21], in which case the correlation length $\xi$ cannot be made infinite although for some systems lying close to $\mathrm{T}_{1}^{\prime \prime}$ and attracted to it (i.e. at the transition temperature), $\xi$ may be very large (because $\mathrm{T}_{1}^{\prime \prime}$ is endless), in which cases one may say that the transition is almost of the second order [22]. Of course, a domain of the first-order phase transition in $S$ was expected out of the usual arguments $[7,6]$. We only better specify the conditions of the first-order transition realization in $S$.

Figure 4 shows the attractive trajectory $\mathrm{T}_{1}^{\prime \prime}$ together with the attractive tricritical line approaching the Gaussian fixed point. The tricritical surface $S_{\mathrm{t}}$ separates the first-order surface $S_{\mathrm{f}}$ from the critical surface $S_{\mathrm{c}}$. Figure 4 also shows that systems lying close to the tricritical surface may still be attracted to the Wilson-Fisher fixed point. In this case the effective exponents may undergo a very retarded crossover to the asymptotic Ising values compared to usual systems corresponding to initial points chosen in the sector $u_{4}>0$ of $S$. Figure 5 illustrates how minus the inverse of (2.2) provides us with different evolutions [calculated from (2.1)] of the effective exponent $\nu_{\text {eff }}(\tau)$ [with $\tau \propto\left(T-T_{\mathrm{c}}\right) / T_{\mathrm{c}}$ ] according to the initial point chosen in $S$. It is worth explaining how we have defined $\nu_{\text {eff }}(\tau)$.

We have seen at the end of section 2 that the quantity (2.2) undergoes a flat extremum (or a flat inflection point) at an RG eigenvalue of $f^{*}$ each time the $\mathrm{RG}$ flow runs along an eigendirection in the vicinity of $f^{*}$. Now it happens that this extremum is less and less flat as one chooses larger and larger values of $\left(u_{2}(0)-u_{2}^{\mathrm{c}}\right)$ (for the eigenvalue $\nu$ ) but it still exists. This provides us with a way of expressing the evolution of an effective exponent $\nu_{\text {eff }}$ when the RG-substitute to $\tau$, namely $\left(u_{2}(0)-\right.$ $\left.u_{2}^{\mathrm{c}}\right) / u_{2}^{\mathrm{c}}$, is varied. Figure 6 shows such an evolution for some initial Hamiltonian (with $u_{4}(0)=4$ ). Notice that for such a Hamiltonian, the extremum disappears before $\nu_{\text {eff }}$ reaches the trivial value $\frac{1}{2}$ (associated with the approach to the trivial high temperature fixed point and to a regular - non critical - behaviour) while in the case of a Hamiltonian initialized close to the tricritical surface, the classical-toIsing crossover is complete (see figure 4). This is because in the latter case the RG 


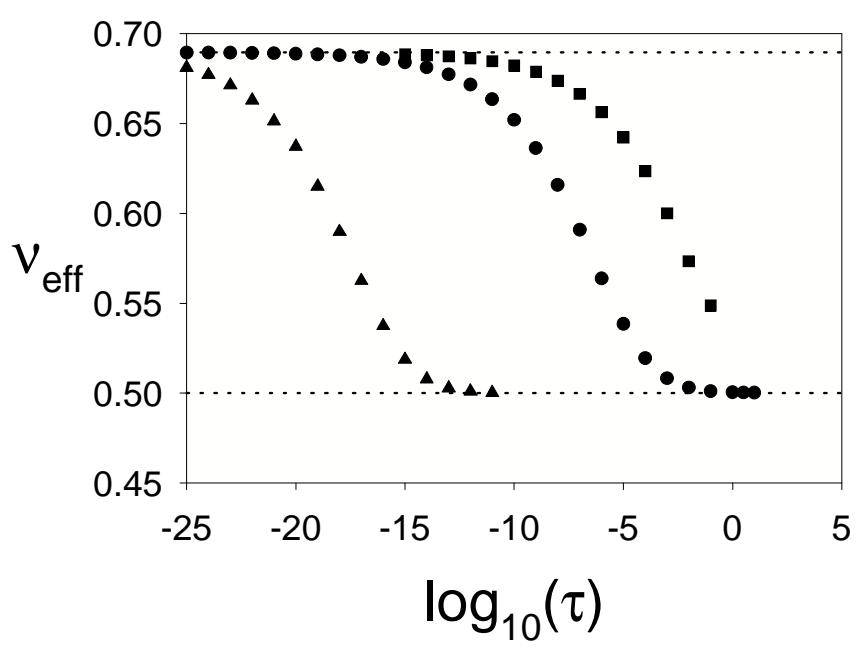

Figure 5. Evolutions of an effective exponent $\nu_{\mathrm{eff}}(\tau)\left[\right.$ with $\left.\tau \propto\left(T-T_{\mathrm{c}}\right) / T_{\mathrm{c}}\right]$ along three different families of RG trajectories (see text for additional details). The full squares indicate the evolution of $\nu_{\text {eff }}(\tau)$ for a family of trajectories initialized in the sector $u_{4}>0$ with $u_{4}(0)=3$ and for various values of $u_{2}(0)$ (the same system at criticality corresponds to the white circle "Simple fluid" of figure 4). When $u_{2}(0) \rightarrow u_{2}^{\mathrm{c}}$ the effective exponent approaches the critical exponent value $\nu \cong 0.69$ compatible with the present study. One observes that the crossover towards the classical value $\frac{1}{2}$ is not complete because $\nu_{\text {eff }}(\tau)$ ceases to make sense before $\tau$ becomes large. This is not the case of the evolution represented by the full circles which correspond to trajectories initialized close to the Gaussian fixed point. In this case the complete crossover reproduces the interpolation between the Gaussian and the Wilson-Fisher fixed points and typically corresponds to the usual answer given by field theory [28]. The third evolution (full triangles) corresponds to a family of Hamiltonian initialized close to the tricritical surface but still attracted to the Wilson-Fisher fixed point. One sees that the classicalto-Ising crossover is complete but highly retarded compared to the two other cases. This is because at criticality, the RG flow is first attracted to the Gaussian fixed point (showing then an apparent classical value of $\nu$ ) before interpolating between the Gaussian and the Wilson-Fisher fixed point.

trajectory comes close to the Gaussian fixed point (and $\nu_{\text {eff }}(\tau)$ has an extremum at $\frac{1}{2}$ ) before approaching $f^{*}$. This reinforces the idea that the so-called classical-to-Ising crossover actually exists only between the Gaussian and Wilson-Fisher fixed points [23].

The same configuration displayed in figure 4 has been also obtained by Tetradis and Litim [24] while studying analytical solutions of an exact RG equation in the local potential approximation for the $O(N)$-symmetric scalar theory in the large $N$ limit. But they were not able to determine "the region in parameter space which results in first-order transitions" [24]. 


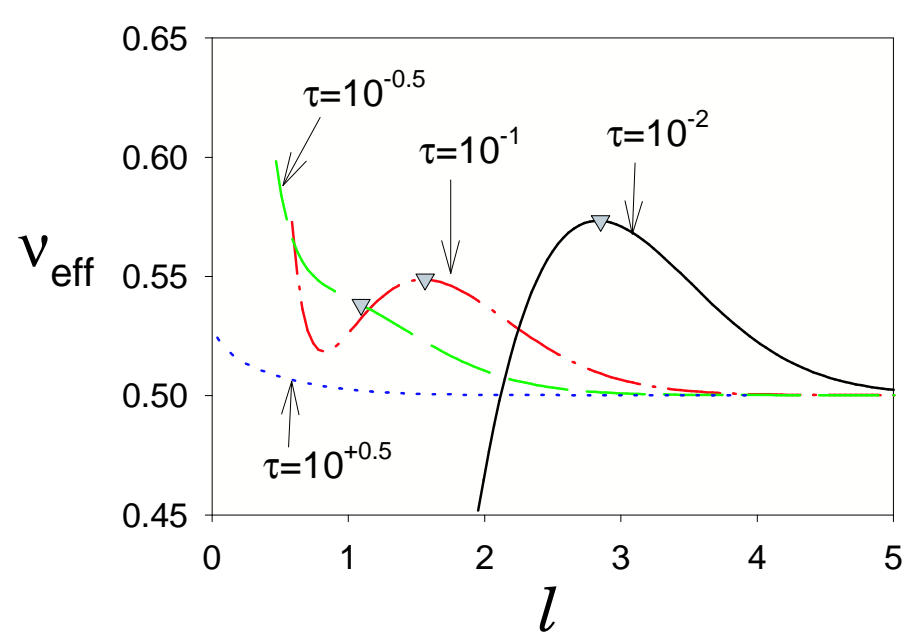

Figure 6. Illustration of the evolution of the extrema $\nu_{\text {eff }}(l)$ [minus the inverse of equation (2.2)] for various values of $\tau=\left(u_{2}(0)-u_{2}^{\mathrm{c}}\right) / u_{2}^{\mathrm{c}}$ and for the family of RG flows initialized at $u_{4}(0)=3$. The extremum (grey triangle) disappears at some not very large value of $\tau$ (about $10^{-0.5}$ ) and does not reach the classical value $\frac{1}{2}$. This induces the partial Ising-to-classical crossover drawn in figure 5 (squares).

\subsection{Case $d=4$}

To decide whether the marginal operator (associated with the eigenvalue equal to zero, i.e. $\lambda_{2}$ in four dimensions, or $\lambda_{3}$ in three dimensions) is relevant or irrelevant, one must go beyond the linear approximation. The analysis is presented in [15] for $d=4$. If one considers a RG flow along $\chi_{2}^{+}$such that $g_{2}(\varphi, l)=c(l) \chi_{2}^{+}(\varphi)$, then one obtains, for small $c$ : $c(l)=c(0)[1-A c(0) l]$ with $A>0$. Hence the marginal parameter decreases as $l$ grows. As it is well known, in four dimensions the marginal parameter is irrelevant. However, if one considers the direction opposite to $\chi_{2}^{+}$(i.e. $\left.\chi_{2}^{-}\right)$then the evolution corresponds to changing $c \rightarrow-c$. This gives, for small values of $c: c(l)=c(0)[1+A|c(0)| l]$ and the parameter becomes relevant. The parameter $c$ is the renormalized $\phi^{4}$ coupling constant $u_{R}$ and it is known that in four dimensions the Gaussian fixed point is IR stable for $u_{R}>0$ but IR is unstable for $u_{R}<0$ [25].

We have verified that the trajectory $\mathrm{T}_{1}^{\prime \prime}$ survives when $d=4$ (contrary to $\mathrm{T}_{1}$, see [2]). That trajectory $\mathrm{T}_{1}^{\prime \prime}$ is a renormalized trajectory on which we could define a continuum limit for the $\varphi_{4}^{4}$-field theory and if the corresponding (perfect) action was positive for all $\varphi$, one could say that the $\phi_{4}^{4}$-field theory with a negative coupling is asymptotically free. Unfortunately, because the $\varphi^{4}$-term is dominant for large $\varphi$ in the vicinity of the origin of $S$ (due to the relevant direction provided by $\chi_{2}^{-}$), the negative sign of the renormalized coupling prevents the path integral to be well defined.

However, because the action to which one refers in the continuum limit (the perfect action) is formal (because it involves an infinite number of parameters and cannot be written down, see [2]) we wonder whether the wrong sign of the action is actually a valid argument to reject the $\varphi_{4}^{4}$-field theory with a negative renormalized coupling. It is worth mentioning that the asymptotically free scalar field theory 
which has recently been considered on a lattice [26] could actually be the $\phi_{4}^{4}$-field theory with a negative coupling to which we refer here.

\section{Acknowledgements}

We dedicate this article to Professor Yukhnovskii in grateful recognition of his efficient and generous help in fostering the Ukrainian-French Symposium held in Lviv in february 1993, with the hope that in the future the contacts between our two communities will further develop.

\section{A. The finite difference method used}

For technical reasons, instead of studying equation (2.1), we consider the differential equation satisfied by $g(\varphi)=f^{\prime}(\varphi)$ (i.e. the second derivative of the potential with respect to the field):

$$
\dot{g}=\frac{K_{d}}{2}\left[\frac{g^{\prime \prime}}{1+g}-\frac{\left(g^{\prime}\right)^{2}}{(1+g)^{2}}\right]+\left(1-\frac{d}{2}\right) \varphi g^{\prime}+2 g .
$$

Starting with a known initial function (at "time" $l=0$ ), we follow its evolution in $S$ by approximating the differential equation (A.1) by finite differences and a two dimensional grid with the uniform spacings $\mathrm{d} y=0.01$ and $\mathrm{d} l=0.000390625$. The finite difference formulas for the derivatives $g^{\prime \prime}$ and $g^{\prime}$ have been chosen with the accuracy $O\left(\mathrm{~d} y^{4}\right)$ :

$$
\begin{aligned}
g^{\prime}(y)= & \frac{8}{12 \mathrm{~d} y}[g(y+\mathrm{d} y)-g(y-\mathrm{d} y)-g(y+2 \mathrm{~d} y)+g(y-2 \mathrm{~d} y)] \\
& +O\left(\mathrm{~d} y^{4}\right), \\
g^{\prime \prime}(y)= & \frac{16}{12 \mathrm{~d} y^{2}}[g(y+\mathrm{d} y)+g(y-\mathrm{d} y)-30 g(y) \\
& -g(y+2 \mathrm{~d} y)-g(y-2 \mathrm{~d} y)]+O\left(\mathrm{~d} y^{4}\right)
\end{aligned}
$$

The evolutionary function $g(y, l)$ is known (calculated) at the discreet set of points $y_{i}=i \cdot \mathrm{d} y$ with $(i \geqslant 0)$ and a maximum value $i_{\max }=82$. (This value is large enough to study the approach to the Wilson-Fisher fixed point with great accuracy but it is too small to precisely study the approach to the Gaussian fixed point.) At each time $l_{k}=k \cdot \mathrm{d} l$, the derivatives are estimated from $g\left(y_{i}, l_{k}\right)=$ $g\left(y_{i}, l_{k-1}\right)+\dot{g}\left(y_{i}, l_{k-1}\right) \cdot \mathrm{d} l$ by using equations (A.2), (A.3) which apply only for $1<i<i_{\max -1}$. For the marginal points $i=0,1$ we use the parity of $g(y)$ [by inserting $g(-n \cdot \mathrm{d} y)=g(n \cdot \mathrm{d} y)$ for $n=1,2$ in equations (A.2), (A.3)]. For the two other marginal points $i=i_{\max -1}, i_{\max }$ of the grid, there is no fixed solution and we shall alternately use the two following conditions [using the obvious abbreviation $g(i)$ instead of $g(i \cdot \mathrm{d} y)]$ :

$$
\begin{aligned}
g^{\prime}(i) & =g^{\prime}(i-1) \\
g^{\prime \prime}(i) & =g^{\prime \prime}(i-1)
\end{aligned}
$$




$$
\begin{aligned}
g^{\prime}(i)=\frac{1}{\mathrm{~d} y} & {\left[\frac{25}{12} g(i)-4 g(i-1)+3 g(i-2)-\frac{4}{3} g(i-3)\right.} \\
& \left.+\frac{1}{4} g(i-4)+O\left(\mathrm{~d} y^{4}\right)\right], \\
g^{\prime \prime}(i)=\frac{1}{\mathrm{~d} y^{2}} & {\left[\frac{915}{244} g(i)-\frac{77}{6} g(i-1)+\frac{107}{6} g(i-2)-13 g(i-3)\right.} \\
& \left.+\frac{61}{12} g(i-4)-\frac{5}{6} g(i-5)\right]+O\left(\mathrm{~d} y^{4}\right) .
\end{aligned}
$$

Condition 2 is more accurate than condition 1 but sometimes leads to strong instabilities which do not appear when we first use condition 1 and then condition 2 after some finite "time" $l_{0}$. The validity of the procedure is tested by, for example, trying to approach a given fixed point (see the main part of the paper).

\section{A.1. Spurious fixed points appearing in approaching the Gaussian fixed point}

In trying to determine the attractive tricritical trajectory (approaching the Gaussian fixed point), we have encountered a spurious twice unstable fixed point lying at some finite and non-negligible distance to the Gaussian fixed point. To understand the origin of this undesirable numerical effect, it is necessary to shortly discuss the solution of the fixed point equation $\dot{f}=0$.

From (2.1) or (A.1), one sees that the fixed point equation is a second order non-linear differential equation and a solution would be parametrized by two arbitrary constants. One of these two constants may easily be determined: since $g^{*}(\varphi)$ is expected to be an even function of $\varphi\left[\mathrm{O}(1)\right.$ symmetry] then $g^{* \prime}(0)=0$ may be imposed. There remains one free parameter, thus a one-parameter family of (nontrivial) fixed points are the solutions to the differential equation. But there is no infinity of physically acceptable fixed points; all but a finite number of the solutions in the family are singular at some $\varphi_{\mathrm{c}}[15,18,27]$. Formally, by requiring the physical fixed point to be defined for all $\varphi$, the acceptable fixed points are limited to the Gaussian fixed point and (for $d=3$ ) to the Wilson-Fisher fixed point.

However, in our study, because we numerically consider the function $g(\varphi)$ in some finite range of values of $\varphi$ (see above: $i_{\max }=82$ ), it appears that in approaching the origin of $S$, infinitely many pseudo-fixed points exist which have there $\varphi_{\mathrm{c}}$-singularity located outside the finite range explicitly considered and there is at least one of them which looks like a tricritical fixed point. When we enlarge the range of $\varphi$ to $i_{\max }=200$, the previously observed nontrivial tricritical fixed point disappears to the benefit of another one located closer to the origin. In conclusion, a larger and larger number of grid-points must be considered as one tries to come closer and closer to the Gaussian fixed point. This particularity together with the slowness of the approach along a marginal direction makes it extremely difficult to come very close to the Gaussian fixed point. 


\section{References}

1. For a review on the exact RG equation, see: Bagnuls C., Bervillier C. Exact renormalization group equations. An introductory review. Preprint hep-th/0002034, 2000 (to appear in Phys. Rep.).

2. Bagnuls C., Bervillier C. Field-theoretic techniques in the study of critical phenomena. // J. Phys. Stud., 1997, vol. 1, p. 366.

3. Zinn-Justin J. Euclidean Field Theory and Critical Phenomena. Third edition, Oxford University Press, 1996.

4. Wilson K.G., Kogut J. The renormalization group and the $\epsilon$-expansion. // Phys. Rep. C, 1974, vol. 12, p. 77.

5. Wilson K.G., Fisher M.E. Critical exponents in 3.99 dimensions. // Phys. Rev. Lett., 1972, vol. 28, p. 240.

6. Aharony A. Critical behaviour of magnets with lattice coupling. // Phys. Rev. B, 1973, vol. 8, p. 4314.

7. Riedel E.K., Wegner F.J. Tricritical exponents and scaling fields. // Phys. Rev. Lett., 1972, vol. 29, p. 349.

8. Brilliantov N.V., Bagnuls C., Bervillier C. Peculiarity of the Coulombic criticality? // Phys. Lett. A, 1998, vol. 245, p. 274.

9. Narayanan T., Pitzer K.S. Mean-field to Ising crossover in ionic fluids. // Phys. Rev. Lett., 1994, vol. 73, p. 3002.

Levin Y., Fisher M.E. Criticality in the hard-sphere ionic fluid. // Physica A, 1996, vol. 225, p. 164.

Stell G. Phase separation in ionic fluids. // J. Phys.: Condens. Matter, 1996, vol. 8, p. 9329.

10. Moreira A.G., Telo da Gama M.M., Fisher M.E. Crossover scales at the critical points of fluids with electrostatic interactions. // J. Chem. Phys., 1999, vol. 110, p. 10058.

11. Hasenfratz P. Perfect actions - From the theoretical background to recent developments. // Prog. Theor. Phys. Suppl., 1998, vol. 131, p. 189.

12. Nicoll J.F., Chang T.S., Stanley H.E. Approximate renormalization group based on the Wegner-Houghton differential generator. // Phys. Rev. Lett., 1974, vol. 33, p. 540; Exact and approximate differential renormalization-group generators. // Phys. Rev. A, 1976, vol. 13, p. 1251.

13. Wegner F.J., Houghton A. Renormalization group equation for critical phenomena. // Phys. Rev. A, 1973, vol. 8, p. 401.

14. Tokar V.I. A new renormalization scheme in the Landau-Ginzburg-Wilson model. // Phys. Lett. A, 1984, vol. 104, p. 135.

15. Hasenfratz A., Hasenfratz P. Renormalization group study of scalar field theories. // Nucl. Phys. B, 1986, vol. 270 [FS16], p. 687.

16. Bagnuls C., Bervillier C. Field theoretical approach to critical phenomena. // Phys. Rev. B, 1990, vol. 41, p. 402.

17. Morris T. R. Three dimensional massive scalar field theory and the derivative expansion of the renormalization group. // Nucl. Phys. B, 1997, vol. 495, p. 477.

18. Felder G. Non-trivial renormalization group fixed points. - In: Proc. Contributed papers of $8^{\text {th }}$ International Congress on Mathematical Physics, Marseille, 1986; Renormalization group in the local potential approximation. // Com. Math. Phys., 1987, vol. 111, p. 101. 
19. Wegner F.J. Corrections to scaling laws. // Phys. Rev. B, 1972, vol. 5, 4529; The critical state, General aspects. - In: Phase Transitions and Critical Phenomena, vol. VI, Ed. by Domb C., Green M.S., Acad. Press, New York, 1976, p. 7.

20. Comellas J., Travesset A. $\mathrm{O}(\mathrm{N})$ models within the local potential approximation. // Nucl. Phys. B, 1998, vol. 498, p. 539.

21. Bak P., Krinsky S., Mukamel D. First-order transitions, symmetry, and the $\epsilon$ expansion. // Phys. Rev. Lett., 1976, vol. 36, p. 52.

Toledano J.-C., Michel L., Toledano P., Brézin E. Renormalization-group study of the fixed points and of their stability for phase transitions with four-component order parameters. // Phys. Rev. B, 1985, vol. 11, p. 7171.

22. Zumbach G. Almost second order phase transitions. // Phys. Rev. Lett., 1993, vol. 71, p. 2421; Phase transitions with $O(n)$ symmetry broken down to $O(n-p)$. // Nucl. Phys. B, 1994, vol. 413, p. 771.

23. Bagnuls C., Bervillier C. Comment on "Nature of crossover between Ising-like and mean-field critical behaviour in fluids and fluid mixtures". // Phys. Rev. Lett., 1996, vol. 76 , p. 4094.

24. Tetradis N., Litim D.F. Analytical solutions of exact renormalization group equations. // Nucl. Phys. B [FS], 1996, vol. 464, p. 492.

25. Symanzik K. A field theory with a computable large-momenta behaviour. // Lett. Nuovo Cimento, 1973, vol. 6, p. 77; Parisi G. Deep inelastic scattering in a field theory with computable large-momenta behaviour. // Lett. Nuovo Cimento, 1973, vol. 7, p. 84; Gawedski K., Kupiainen A. Non-trivial continuum limit of a $\phi_{4}^{4}$ model with negative coupling constant. // Nucl. Phys. B, 1985, vol. 257 [FS14], p. 474; Asymptotic freedom beyond perturbation theory. - In: Critical Phenomena, Random Systems, Gauge Theories, Ed. by Osterwalder K. and Stora R., Elsevier Science Pub. B.V., 1986, p. 185; See also, 't Hooft G. The birth of asymptotic freedom. // Nucl. Phys. B, 1985, vol. 254, p. 11.

26. Langfeld K., Reinhardt H. Asymptotic freedom in a scalar field theory on the lattice. // Mod. Phys. Lett. A, 1998, vol. 13, p. 2495.

27. Filippov A.E., Breus S.A. On the physical branch of the exact (local) RG equation. // Phys. Lett. A, 1991, vol. 158, p. 300; Filippov A.E. Solution of exact (local) renormalization-group equation. // Theor. Math. Phys., 1992, vol. 91, p. 551; Breus S.A., Filippov A.E. Study of a local RG approximation. // Physica A, 1993, vol. 192, p. 486; Morris T.R. Derivative expansion of the exact renormalization group. // Phys. Lett. B, 1994, vol. 329, p. 241; On truncations of the exact renormalization group. // Phys. Lett. B, 1994, vol. 334, p. 355.

28. Bagnuls C., Bervillier C. Nonasymptotic critical behaviour from field theory at $d=3$ : The disordered-phase case. // Phys. Rev. B, 1985, vol. 32, p. 7209. 


\title{
Області ренормалізаційної групи скалярного гамільтоніану
}

\author{
К.Банюльс ${ }^{1}$, К.Бервільє ${ }^{2}$ \\ 1 Відділ фізики конденсованого стану, Саклє, Франція \\ 2 Відділ теоретичної фізики, Саклє, Франція
}

Отримано 9 березня 2000 р.

Використовуючи наближення локального потенціалу точного рівняння ренормалізаційної групи (РГ), ми показуємо різні області значень параметрів О(1) симетричного скалярного гамільтоніану. У трьох вимірах додатково до звичайної критичної поверхні $S_{\text {с }}$ (область притягання фіксованої точки Вільсона-Фішера), ми явно показуємо існування області фазового переходу першого ряду $S_{\mathrm{f}}$, відокремленої від $S_{\text {c }}$ трикритичною поверхнею $S_{\mathrm{f}}$ (область притягання гаусової фіксованої точки). $S_{\mathrm{f}}$ і $S_{\mathrm{c}} \in$ дві різні області відштовхування для гаусової фіксованої точки, а $S_{\mathrm{f}}$ не $є$ в ділянці притягання фіксованої точки. $S_{\mathrm{f}}$ характеризується нескінченою ренормалізованою траєкторією, яка повністю лежить в області негативних значень констант взаємодії $\varphi^{4}$. Ця ренормалізована траєкторія також існує в чотирьох вимірах, роблячи гаусову фіксовану точку в ультрафіолетовій області стабільною (і ренормалізовану теорію поля $\varphi^{4}$ асимптотично вільною, але з неправильним знаком ідеальної дії). Ми також показуємо, що дуже запізнений кросовер від класичної до ізінгівської поведінки може існувати у трьох вимірах (фактично нижче чотирьох вимірів). Це може бути поясненням для неочікуваної класичної критичної поведінки, яка спостерігається в деяких іонних системах.

Ключові слова: ренормалізаційна група, критичний, трикритичний, фазовий перехід першого роду, кросовер

PACS: 05.10.Cc, 05.70.Jk, 11.10. Hi, 61.20.Qg 
